\title{
ISOLAMENTO E IDENTIFICAÇÃO DA MORINA EM MEL BRASILEIRO DE Apis mellifera
}

\author{
Regina Lucia Pelachim Lianda e Rosane Nora Castro* \\ Departamento de Química, Instituto de Ciências Exatas, Universidade Federal Rural do Rio de Janeiro, Antiga Rodovia Rio-São \\ Paulo, BR 465, km 07, 23.890-000 Seropédica - RJ, Brasil \\ Recebido em 23/10/07; aceito em 27/3/08; publicado na web em 1/9/08 \\ ISOLATION AND IDENTIFICATION OF MORIN IN BRAZILIAN HONEY FROM Apis mellifera. The flavonoid fraction \\ was purified by a combination of chromatography on Amberlite XAD-2 and preparative silica gel TLC. Morin (3, 5, 7, 2', 4'- \\ pentahydroxyflavone) was the only flavonol found in honey from Brazilian Citrus sp.. The structure of morin was determined on the \\ basis of $\mathrm{UV}$ and ${ }^{1} \mathrm{H}$ and ${ }^{13} \mathrm{C}$ NMR spectral data together with literature references. This is the first report on the isolation of morin \\ from Brazilian Citrus honey.
}

Keywords: Brazilian honey; morin; Apis mellifera.

\section{INTRODUÇÃO}

O mel é uma mistura de substâncias produzida pelas abelhas (no Brasil, Apis mellifera) a partir da coleta do néctar das flores ou de secreções de partes de plantas ou de excreções de insetos que sugam partes das plantas, e que se transformam e combinam com as substâncias específicas de seus próprios organismos. Apesar de o mel ser basicamente uma solução saturada de açúcares e água, seus outros componentes, aliados às características da fonte floral que o originou, conferem-lhe um alto teor nutritivo e propriedades medicinais. Além dos açúcares destacam-se os aminoácidos, enzimas, proteínas, sais minerais, vitaminas e substâncias fenólicas. ${ }^{1}$ Apesar dos carboidratos encontrados no mel serem objetos de diversas revisões, ${ }^{2}$ outros componentes presentes não foram extensivamente estudados, como por exemplo, os compostos fenólicos. Seu aroma, paladar, coloração, viscosidade e propriedades medicinais estão diretamente relacionadas com a fonte de néctar que o originou e também com a espécie de abelha que o produziu. ${ }^{1}$ A composição química do mel depende, em grande parte, das espécies vegetais visitadas pelas abelhas, natureza do solo, raça das abelhas e condições climáticas. Levando-se em conta a grande variedade do clima e da flora, extremamente ricas, encontradas no Brasil, a produção de mel e sua composição merecem especial atenção. Características físico-químicas, tais como, consistência, cor, odor, sabor e aromas, geralmente mantêm-se constantes e ocorrem somente em méis chamados monoflorais ou uniflorais, isto é, aqueles procedentes do néctar de uma espécie vegetal predominante, por exemplo, mel de laranjeiras, mel de eucalipto, etc. Estes méis são cada vez mais procurados pelo consumidor. ${ }^{3}$

As substâncias fenólicas contidas no mel podem ser oriundas do néctar coletado pelas abelhas, do pólen ou, ainda, da própolis. Muitas dessas substâncias, principalmente os ácidos fenólicos e flavonóides, são conhecidas por apresentarem propriedades farmacológicas e tornaram-se alvos de estudo, devido a sua conhecida ação sobre numerosos processos fisiológicos no corpo, podendo beneficiar o coração, veias, fígado, sistema imunológico, rins, musculatura e sistema nervoso. ${ }^{4,5}$ Das várias propriedades terapêuticas atribuídas a essas substâncias, podem se destacar os efeitos antioxidantes, antibacterianos, antialérgicos, antiinflamatórios, etc. ${ }^{6}$

Neste trabalho é descrito o isolamento e a determinação estru-

*e-mail: nora@ufrrj.br tural da morina (1), oriunda de uma amostra de mel de laranjeiras (monofloral).

\section{PARTE EXPERIMENTAL}

\section{Procedimentos experimentais gerais}

Os espectros obtidos na região do ultravioleta-visível foram registrados em espectrofotômetro Shimadzu, modelo UV mini 1240, utilizando como solvente metanol grau espectroscópico.

Os espectros de ressonância magnética nuclear (RMN) foram obtidos em espectrômetro Brücker, modelo AC-200, operando a $200 \mathrm{MHz}$ para RMN ${ }^{1} \mathrm{H}$ e a $50 \mathrm{MHz}$ para $\mathrm{RMN}{ }^{13} \mathrm{C}$, com as amostras solubilizadas em DMSO- $d 6$ e usando o tetrametilsilano (TMS) como padrão interno. Os deslocamentos químicos $(\delta)$ foram obtidos em parte por milhão (ppm) e as constantes de acoplamento $(J)$ foram medidas em Hertz $(\mathrm{Hz})$.

Nas análises por cromatografia em camada delgada (CCD) foram utilizadas cromatofolhas de alumínio contendo gel de sílica $60 \mathrm{~F}_{254}$ (Merck), e revelação com luz ultravioleta ( 254 e $366 \mathrm{~nm}$ ) e/ou nebulização com solução $1 \%$ de $\mathrm{AlCl}_{3}$ em etanol, seguida de aquecimento. Para a análise dos flavonóides não-glicosilados o sistema de solvente usado foi uma mistura de hexano:acetato de etila:ácido fórmico (19:20:1) e para os flavonóides glicosilados o sistema de solvente usado foi uma mistura de clorofórmio:metanol:água:ácido fórmico (30:18:1:1).

$\mathrm{Na}$ separação por cromatografia em camada delgada preparativa (CCDP) foi utilizada placa de vidro $20 \times 20 \mathrm{~cm}$ recoberta com gel de sílica com indicador de fluorescência $\left(\mathrm{F}_{254}\right.$, Merck), e para a eluição uma mistura de hexano:acetato de etila:ácido fórmico (15:24:1). Todos os solventes utilizados foram de pureza analítica ou grau espectroscópico.

Os padrões utilizados para as análises por cromatografias de camada delgada foram sempre de grau de pureza elevado (99\%). Os flavonóides quercetina, morina, hesperidina, rutina foram obtidos da Merck. A miricetina foi isolada no Laboratório Químico de Produtos Naturais (DEQUIM-UFRRJ). As soluções dos padrões foram preparadas a $1 \%$ em metanol (grau espectroscópico) e utilizadas como solução estoque padrão.

\section{Amostra de mel}

A amostra de mel de laranjeiras (Citrus sp, codificada de RLL06) foi coletada da região de Mata Atlântica e adquirida diretamente do 
apicultor, em março de 2004, na cidade de Taubaté-SP. A identificação da fonte floral foi baseada nas propriedades sensoriais, tais como, aroma, paladar e cor do mel; a localização das colméias; a estação na qual o mel foi coletado e vegetação nas proximidades. A amostra foi estocada na geladeira a $4{ }^{\circ} \mathrm{C}$ até o momento da análise, a fim de evitar qualquer tipo de alteração.

\section{Extração e isolamento das substâncias fenólicas do mel}

As substâncias fenólicas foram extraídas do mel segundo adaptação ${ }^{7}$ à metodologia descrita na literatura. ${ }^{8} \mathrm{~A}$ amostra de mel de laranjeiras RLL06 (50 g) foi misturada com $250 \mathrm{~mL}$ de água destilada, acidificada a pH 2 com $\mathrm{HCl}$ concentrado e agitada com agitador magnético, a temperatura ambiente, até completa dissolução. A amostra fluida foi, em seguida, filtrada através de algodão para eliminar possíveis partículas suspensas. O filtrado foi então agitado com cerca de $75 \mathrm{~g}$ de Amberlite XAD-2 (tamanho do poro $9 \mathrm{~nm}$ e partícula 0,3-1,2 mm, Fluka), e, em seguida, empacotado em uma coluna de vidro ( 45 x 3,5 $\mathrm{cm}$ ). A coluna foi então lavada primeiramente com uma solução ácida ( $\mathrm{pH} 2$ com HCl concentrado, $100 \mathrm{~mL}$ ), e em seguida com água destilada $(150 \mathrm{~mL})$ para remover todos os açúcares e outros compostos polares, enquanto as substâncias fenólicas presentes no mel permaneceram na coluna. A fração fenólica adsorvida na coluna foi então eluída com metanol $(\sim 250 \mathrm{~mL})$. O extrato metanólico obtido foi concentrado à pressão reduzida em um evaporador rotatório a $40{ }^{\circ} \mathrm{C}$. O resíduo foi dissolvido em $15 \mathrm{~mL}$ de água destilada, e extraído com acetato de etila ( 5 x $10 \mathrm{~mL}$ ). As fases orgânicas foram reunidas, secas com sulfato de sódio anidro, concentradas a pressão reduzida, e dissolvidas em metanol para serem analisadas por CCD e depois aplicadas em CCDP.

O extrato de acetato de etila obtido da amostra RLL06 (70 mg) foi purificado através de CCDP e eluído com hexano:acetato de etila:ácido fórmico (15:24:1). Após a eluição a placa foi visualizada na lâmpada a 254 e $366 \mathrm{~nm}$, respectivamente, para auxiliar a retirada da substância de interesse da placa. O material foi raspado da placa, misturado com $20 \mathrm{~mL}$ de metanol, deixado agitar por 30 min e filtrado em papel de filtro. Em seguida, o metanol foi evaporado até a secura e forneceu 5,6 mg do flavonóide 1 (morina, Figura 1) como um sólido de coloração amarelo-castanho, que apresentou característica de uma substância pura por CCD.<smiles>O=c1c(O)c(-c2ccc(O)cc2O)oc2cc(O)cc(O)c12</smiles>

Figura 1. Substância isolada do mel de laranjeira de Apis mellifera

\section{RESULTADOS E DISCUSSÃO}

A amostra do mel de laranjeiras (RLL06) foi submetida ao fracionamento em coluna de Amberlite XAD-2 com metanol. O extrato metanólico foi analisado por CCD e as frações de similares fatores de retenção $\left(\mathrm{R}_{\mathrm{f}}\right)$, comparadas com o padrão autêntico da morina, foram reunidas. A análise destas frações revelou claramente a presença do flavonol 1 através da observação do mesmo fator de retenção $\left(\mathrm{R}_{\mathrm{f}}=\right.$ $0,44)$, bem como pelo aparecimento da coloração amarelo fluorescente a $366 \mathrm{~nm}$, após revelação com $\mathrm{AlCl}_{3}-\mathrm{EtOH}(1 \%)$ e aquecimento, sendo isto um indicativo para flavonóides com grupo hidroxila no carbono cinco. ${ }^{9}$
A partir destas observações foi realizado o isolamento da morina (1) por cromatografia de camada fina preparativa e a sua estrutura foi identificada com base nas análises dos espectros de UV e RMN.

A análise dos espectros de absorção no ultravioleta, com varredura na faixa de $\lambda 200-500 \mathrm{~nm}$, do padrão em comparação com a substância 1 isolada, forneceu considerações importantes sobre a posição dos grupamentos hidroxilas presentes no esqueleto flavônico, particularmente pela análise da banda I. A análise do espectro no UV de $1 \mathrm{em}$ metanol permitiu observar dois máximos de absorção em $\lambda_{\text {máx }} 256$ e $368 \mathrm{~nm}$ que caracterizam a banda II (absorção do anel A, sistema benzoíla) e a banda I (anel B, sistema cinamoíla), respectivamente, sendo compatível com um flavonol. ${ }^{9}$

$\mathrm{O}$ uso de cloreto de alumínio $\left(\mathrm{AlCl}_{3}\right)$ auxiliou na determinação espectrofotométrica da presença de hidroxilas "peri" e orto, características de alguns flavonóides, por formar complexos estáveis, e promover desvio para maiores comprimentos de onda e uma intensificação da absorção. ${ }^{9}$ Desta forma, a determinação das estruturas de flavonóides torna-se possível, pois ao formar complexos com o cloreto de alumínio possibilitam a sua diferenciação das outras substâncias fenólicas que estão presentes no mel, principalmente os ácidos fenólicos, pois estes, apesar de formarem complexos com cloreto de alumínio, absorvem em comprimentos de onda menores. ${ }^{10}$

Nota-se que com a adição de $\mathrm{AlCl}_{3}$ formou-se um complexo flavonóide-Al que absorveu em comprimento de onda bem maior que o flavonóide sem a presença deste aditivo. A adição de $\mathrm{AlCl}_{3}$ provocou um deslocamento batocrômico nas bandas I e II, onde ocorreu a formação de um anel de seis membros estável através do átomo de alumínio com a hidroxila em C-5 e o grupo carbonila em C-4, resistente à ação de $\mathrm{HCl}$. Já uma hidroxila em C-3, por se tratar de uma hidroxila tipo enólica, pode formar também ligação de hidrogênio intramolecular, porém em anel de cinco membros menos resistente à adição de $\mathrm{HCl}$. Logo após a adição da solução de $\mathrm{HCl}$, observou-se que o complexo que envolveu o grupo carbonila em C-4 e o grupo hidroxila em C-5 apresentou-se estável pela quase manutenção do comprimento de onda da banda II (anel A), porém o complexo formado com a hidroxila em $\mathrm{C}-3$ revelou deslocamento hipsocrômico da banda I (anel B) em relação à amostra com $\mathrm{AlCl}_{3}$. A Figura 2 apresenta os espectros da amostra $\mathbf{1}$ em metanol, após a adição de $\mathrm{AlCl}_{3}$ e depois da adição de solução $15 \%$ de $\mathrm{HCl}$, confirmando ser a estrutura da $3,5,7,2^{\prime}, 4^{\prime}$ - pentaidroxiflavona ou morina (Figura 2).

O flavonol morina (1) foi identificado também, através das análises de $\mathrm{RMN}$ de ${ }^{1} \mathrm{H} \mathrm{e}{ }^{13} \mathrm{C}$ unidimensional em comparação com os

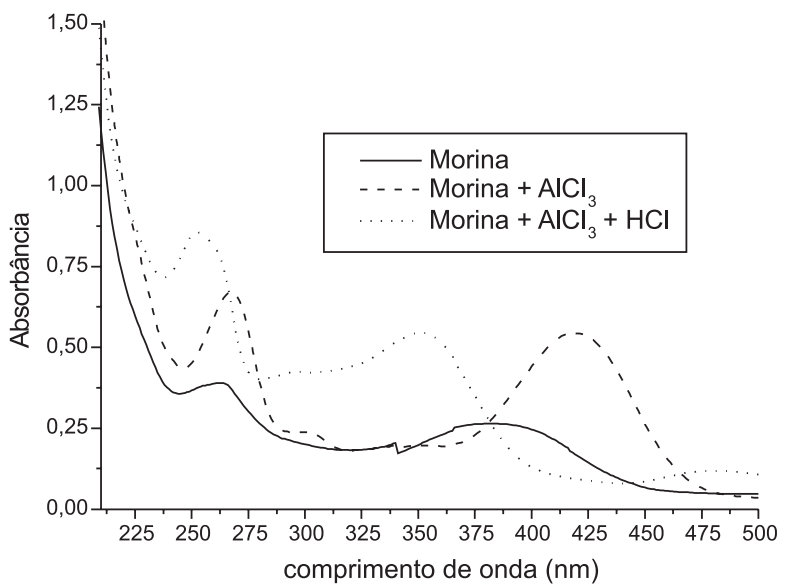

Figura 2. Espectros de absorção no $U V(200-500 \mathrm{~nm})$ para: morina $1 \mathrm{em}$ metanol (-); morina $\underline{\mathbf{1}} \mathrm{com} \mathrm{AlCl}_{3}(--)$ e após adição de $\mathrm{HCl}(\ldots)$ 
dados descritos na literatura. ${ }^{11-13}$

A análise do espectro de $\mathrm{RMN}{ }^{1} \mathrm{H}$ da substância 1 permitiu identificar sinal de hidroxila $\delta_{\mathrm{H}} 12,61 \mathrm{ppm}$, confirmando a presença do hidrogênio do grupo hidroxila, localizada no átomo de carbono C-5, envolvido em ligação de hidrogênio intramolecular com a carbonila em C-4. Na região de deslocamento químico dos hidrogênios aromáticos foram observados dois dubletes centrados em $\delta_{\mathrm{H}} 6,15$ $\operatorname{ppm}(1 \mathrm{H}, \mathrm{d}, J=2,0 \mathrm{~Hz}, \mathrm{H}-6)$ e $\delta_{\mathrm{H}} 6,27 \mathrm{ppm}(1 \mathrm{H}, \mathrm{d}, J=2,0 \mathrm{~Hz}, \mathrm{H}-8)$ presente no anel A. Além desses sinais, foram observados outros três sinais em $\delta_{\mathrm{H}} 6,35 \mathrm{ppm}(1 \mathrm{H}, \mathrm{dd}, J=2,2 \mathrm{~Hz}, \mathrm{H}-5$ '-3' e $J=8,4$ Hz, H-5'-6'); 6,41 ppm (1H, d, $J=2,2 \mathrm{~Hz}, \mathrm{H}$ 3') e 7,21 (1H, d, $J$ $=8,4 \mathrm{~Hz}, \mathrm{H}-6$ ') relacionados ao sistema $\mathrm{ABC}$ do anel aromático $\mathrm{B}$ trissubstituído (Tabela 1).

Tabela 1. Dados de RMN ${ }^{1} \mathrm{He} \mathrm{e}^{13} \mathrm{C}$ da substância 1 (DMSO- $d_{6}$ ) comparados com os dados da literatura para a morina ${ }^{12}$. Deslocamentos químicos em $\delta_{\mathrm{H}}$ e $\delta_{\mathrm{C}}(\mathrm{ppm})$ e constantes de acoplamento ( $J$, entre parênteses) em $\mathrm{Hz}$

\begin{tabular}{lcccc}
\hline \multicolumn{4}{c}{ Substância $\underline{3}$} & \multicolumn{2}{c}{ Morina } \\
& $\delta_{\mathrm{H}}$ & $\delta_{\mathrm{C}}$ & $\delta_{\mathrm{H}}$ & $\delta_{\mathrm{C}}$ \\
\hline $\mathbf{2}$ & & 148,2 & & 149,0 \\
$\mathbf{3}$ & & 139,8 & & 136,3 \\
$\mathbf{4}$ & & 176,4 & & 176,2 \\
$\mathbf{5}$ & $\mathrm{OH}-12,61(\mathrm{~s})$ & 161,1 & $\mathrm{OH}-12,83(\mathrm{~s})$ & 160,9 \\
$\mathbf{6}$ & $6,15(\mathrm{~d} ; 2,0)$ & 98,4 & $6,17(\mathrm{~d} ; 1,7)$ & 98,0 \\
$\mathbf{7}$ & & 164,03 & & 163,7 \\
$\mathbf{8}$ & $6,27(\mathrm{~d} ; 2,0)$ & 93,8 & $6,28(\mathrm{~d} ; 1,7)$ & 93,4 \\
$\mathbf{9}$ & & 156,8 & & 156,8 \\
$\mathbf{1 0}$ & & 105,1 & & 103,6 \\
$\mathbf{1}$ & & 110,8 & & 109,3 \\
$\mathbf{2}$ & & 156,9 & & 156,9 \\
3' & $6,41(\mathrm{~d} ; 2,2)$ & 102,1 & $6,38(\mathrm{~d}, J=2,1)$ & 103,0 \\
$\mathbf{4}$ & & 159,7 & & 160,5 \\
$\mathbf{5}$ & $6,35(\mathrm{dd} ; 8,4 ; 2,2)$ & 107,7 & $6,33(\mathrm{dd} ; 8,3 ; 2,1)$ & 106,8 \\
$\mathbf{6}$, & $7,21(\mathrm{~d} ; 8,4)$ & 128,9 & $7,22(\mathrm{~d} ; 8,3)$ & 131,7 \\
\hline
\end{tabular}

multiplicidade: $\mathrm{s}=$ singleto; $\mathrm{d}=$ dubleto; $\mathrm{dd}=$ duplo dubleto

A substância 1 exibiu no espectro de $\mathrm{RMN}{ }^{13} \mathrm{C}$ quinze sinais na região de carbonos $\mathrm{sp}^{2}$, compatíveis com a unidade $\mathrm{C}_{6}-\mathrm{C}_{3}-\mathrm{C}_{6}$ característica de flavonóides. Além disso, foi possível identificar a presença de cinco carbonos contendo grupos hidroxilas (C-3, C-5, C-7, C-2' e C-4'). O deslocamento químico do sinal da carbonila a $\delta_{C} 176,4$ ppm foi influenciado pela ligação intramolecular com o hidrogênio do grupo OH na posição 5 (hidroxila "peri”). A comparação dos dados espectroscópicos obtidos para a substância $\mathbf{1}$ com os registrados na literatura permitiu a identificação deste constituinte como sendo a morina. ${ }^{11-13}$ (Tabela 1).

Os deslocamentos químicos observados nos espectros de RMN de ${ }^{1} \mathrm{He}{ }^{13} \mathrm{C}$, bem como a análise no UV permitiram determinar a estrutura de 1 como 3,5,7,2',4'-pentaidroxiflavona ou morina.

Embora tenha sido relatada na literatura a presença da flavanona hesperitina em mel europeu de laranjeiras, ${ }^{14}$ até então, a morina nunca havia sido isolada e identificada em méis europeus, apesar de ser um flavonóide largamente distribuído entre frutas e vegetais. De acordo com relatos da literatura, sabe-se que para os méis de laranjeiras, a análise polínica não é simples como nos casos dos outros méis uni- florais, devido ao fato de que a quantidade de pólen presente neste mel é pequena e geralmente muito variada. ${ }^{15}$ Diferentes espécies de plantas produzem quantidades de pólen diferentes, que podem variar de estação para estação. Sendo assim, o mel pode apresentar os grãos de pólen provenientes, na sua maior parte, das plantas fornecedoras de néctar, as chamadas plantas nectaríferas; bem como das chamadas plantas poliníferas que, além de pouco néctar, fornecem bastante pólen. Certa percentagem do pólen no mel pode ainda ser proveniente de plantas anemófilas, isto é, cujas flores não produzem néctar, somente pólen o qual é disperso pelo vento e as abelhas podem aproveitá-lo como fonte de proteínas. ${ }^{3} \mathrm{Na}$ prática não é possível se obter um mel 100\% monofloral, ou seja, isento de grãos de pólen de outros espécimes.

Uma possível razão para se justificar a presença da morina em mel Citrus sp, que foi coletado na região de Mata Atlântica, pode ser em virtude da existência de várias espécies nativas e abundantes na região de coleta do mel; como no caso a presença de Chlorophora tinctoria (L.) Gaudich, que já foi estudada e revelou a presença da morina em sua composição química. ${ }^{16}$

Este trabalho descreve pela primeira vez o isolamento e a identificação da morina em mel brasileiro de laranjeiras. Outros méis de laranjeiras da região da Mata Atlântica já haviam sido estudados e revelaram a presença desta substância por cromatografia líquida de alta eficiência. ${ }^{7}$

\section{AGRADECIMENTOS}

Aos órgãos de fomento CAPES, FAPERJ, CNPq pela bolsa concedida e pelo auxílio financeiro para o desenvolvimento dessa pesquisa, e ao Prof. Dr. M. G. de Carvalho pelo fornecimento do padrão de miricetina.

\section{REFERÊNCIAS}

1. Crane, E.; O livro do mel, $2^{\mathrm{a}}$ ed., Nobel: São Paulo, 1985.

2. Siddiqui, I. R.; Advanc. Carbohydr. Chem. Biochem. 1970, 25, 285; Mendes, E.; Proença, E. B.; Ferreira, I. M. P. L. V. O.; Ferreira, M. A.; Carbohydr. Polym. 1998, 37, 219.

3. Barth, O. M.; Scientia Agrícola 2004, 61, 342.

4. Muñoz, O.; Copaja, S.; Speisky, H; Peña, R. C.; Montenegro, G.; Quim. Nova 2007, 30, 841.

5. Manach, C.; Scalbert, A.; Morand, C.; Rémésy, C.; Jiménez, L.; Am. J. Clin. Nutr. 2004, 79, 727; Yao, L.; Jiang, Y.; Singanusong, R.; Datta, N.; Raymont, K.; Food Chem. 2004, 86, 169; Martos, I.; Ferreres, F.; Tomás-Barberán, F. A.; J. Agric. Food Chem. 2000, 48, 1498; Martos, I.; Cossentini, M.; Ferreres, F.; Tomás-Barberán, F. A.; J. Agric. Food Chem. 1997, 45, 2824; Tomás-Barberán, F. A.; Ferreres, F.; Blázquez, A.; Garcia-Viguera, C.; Tomás-Lorente, F.; J. Chromatogr. 1993, 634, 41; Ferreres, F.; Ortiz, A.; Silva, C.; Garcia-Viguera, C.; TomásBarberán, F. A.; Tomás-Lorente, F.; Z. Lebensm. Unters. Forsch. 1992, 194, 139; Sabatier, S.; Amiot, M. J.; Tacchini, M.; Aubert, S.; J. Food Sci. 1992, 57, 773; Amiot, M. J.; Aubert, S.; Gonnet, M.; Tacchini, M.; Apidologie 1989, 20, 115.

6. Nazime, M.; Aykut, G.; Celik, A.; Katircioglu, H.; Nat. Prod. Res. 2007, 21, 187; Brudzynski, K.; Can. J. Microbiol. 2006, 52, 1228; Kampa, M.; Alexaki, V. I.; Notas, G.; Nifli, A. P.; Nistikaki, A.; Hatzoglou, A.; Bakogeorgou, E.; Kouimtzoglou, E.; Blekas, G.; Boskou, D.; Gravanis, A.; Castanas, E.; Cancer Res. 2004, 6, 63; Aljadi, A. M.; Kamaruddin, M. Y.; Food Chem. 2004, 85, 513; Aljadi, A. M.; Kamaruddin, M. Y.; Turk. J. Med. Sci. 2003, 33, 229; Campos, M. G.; Webby, R. F.; Markham, K. R.; Mitchell, K. A.; Da Cunha, A. P.; J. Agric. Food Chem. 2003, 51, 742; Miorin, P. L.; Junior, N. C. L.; Custodio, A. R.; Bretz, W. A.; Marcucci, M. C.; J. Appl. Microbiol. 2003, 95, 913; Prior, R. L.; Am. J. 
Clin. Nutr. 2003, 78, 570S; Al-Mamary, M.; Al-Meeri, A.; Al-Habori, M.; Nutr. Res. 2002, 22, 1041; Nijveldt, R. J.; Nood, E. V.; Hoorn, D. E. C. V.; Boelens, P. G.; Norren, K. V.; Leeuwen, P. A. M. V.; Am. J. Clin. Nutr. 2001, 74, 418.

7. Lianda, R. L. P.; Dissertação de Mestrado, Universidade Federal Rural do Rio de Janeiro, Brasil, 2004

8. Baltrusaityté, V.; Venskutonis, P. R.; Ceksteryté, V.; Food Chem. 2007, 101, 502; Fiorani, M.; Accorsi, A.; Blasa, M.; Diamantini, G.; Piatti, E.; J. Agric. Food Chem. 2006, 54, 8328; Tomás-Barberán, F. A.; Martos, I.; Ferreres, F.; Radovic, B. S.; Anklam, E.; J. Sci. Food Agric. 2001, 81, 485; Ferreres, F.; Tomás-Barberán, F. A.; Giner, J. M.; J. Sci. Food Agric. 1994, 65, 371; Martos, I.; Ferreres, F.; Tomás-Barberán, F. A.; J. Agric. Food Chem. 2000, 48, 1498.

9. Mabry, T J.; Markham, K R.; Thomas, M B.; The Systematic Identification of Flavonoids, University of Texas, Austin: New York, 1970.

10. Braz-Filho, R.; Tese de Doutorado, Universidade Federal Rural do Rio de Janeiro, Brasil, 1971; Markhan, K. R.; Techniques of Flavonoid Identification, Academic Press: London, 1982; Harborne, J. B.; The Handbook of Natural Flavonoids, Wiley: Chichester, 1999.

11. Da Silva, T. M. S.; Tese de Doutorado, Universidade Federal Rural do Rio de Janeiro, Brasil, 2002.

12. Niassy, B.; Um, B. H.; Lobstein, A.; Weniger, B.; Koné, M.; Anton, R.; C. R. Chimie 2004, 7, 993.

13. Agrawal, P. K.; Carbon-13 NMR of Flavonoids: Studies in Organic Chemistry 39, Elsevier: Amsterdan, 1989.

14. Ferreres, F.; Tomás-Barberán, F. A.; Giner, J. M.; J. Sci. Food Agric. 1994, 65, 371. Ferreres, F.; Garcia-Viguera, C.; Tomás-Barberán, F. A.; Tomás-Lorente, F.; J. Sci. Food Agric. 1993, 61, 121.

15. Serra Bonvehi, J.; Gómez-Pajuelo, A.; Gonell-Galindo, F.; Alimentaria 1987, 185,61 .

16. Wu, T. W; Zeng, L. H.; Wu, J. ; Fung, K. P.; Biochem. Pharmacol. 1994, 47, 1099. 